\title{
A simple motion differential game with different constraints on controls and under phase constraint on the state of the evader
}

\begin{abstract}
We consider a simple motion pursuit differential game of one pursuer and one evader. Control of the pursuer is subjected to integral constraint, and that of the evader is subjected to geometric constraint. More precisely, value of control parameter of the evader belongs to a given convex subset of $\mathbb{R} n$. Pursuit is completed if the evader becomes in 1 vicinity of the pursuer. Sufficient conditions of completion of pursuit are obtained.
\end{abstract}

Keyword: Simple motion; Game; Pursuer; Evader; Control of the pursuer 